\title{
Distribuição horizontal da macrofauna bentônica na praia do Cassino, extremo sul do Brasil
}

\author{
Luciano Peyrer das Neves, Pedro de Sá Rodrigues da Silva \& Carlos Emílio Bemvenuti
}

Fundação Universidade Federal do Rio Grande (FURG), Departamento de Oceanografia, Laboratório de Ecologia de Invertebrados Bentônicos. Av. Itália Km 8. Caixa Postal 474, 96201-900, Rio Grande, RS, Brasil. (peyrer@ig.com.br; pedrobio@hotmail.com; docbemve@furg.com)

\begin{abstract}
Longshore distribution of the benthic macrofauna on Cassino beach, southernmost Brazil. The longshore distribution of the benthic macrofauna on Cassino beach, Rio Grande do Sul, Brazil were studied during one year (from June 2004 to May 2005) on a monthly basis sampling. Three sites were chosen $50 \mathrm{~m}$ apart from each other. On each site three transects, $2 \mathrm{~m}$ apart from each other were anchored. Each transect extended from the base of the primary dunes to approximately $1 \mathrm{~m}$ depth into the infralittoral. The distances to the sampling levels in each transect were $20 \mathrm{~m}$ to the upper limit of the swash zone, from which the distances were of $10 \mathrm{~m}$. The longshore variation of major species and community was highlighted in a $50 \mathrm{~m}$ to $100 \mathrm{~m}$ range in some occasions. This variation occurred, probably, due to the water level elevation because the meteorological factors.
\end{abstract}

KEYWORDS. Sandy beaches, benthic community, spatial-temporal distribution, richness.

\begin{abstract}
RESUMO. A distribuição horizontal da macrofauna bentônica na praia do Cassino, Rio Grande do Sul, Brasil foi estudada durante o período de um ano (junho 2004 a maio de 2005) com base em coletas mensais. Foram escolhidos três locais, com 50 m de distância um do outro, sendo que em cada local foram fundeadas três transversais $2 \mathrm{~m}$ equidistantes. Cada transversal estendeu-se da base das dunas primárias até aproximadamente $1 \mathrm{~m}$ de profundidade no infralitoral. A distância dos níveis de coleta em cada transversal foi de $20 \mathrm{~m}$ até o limite superior da zona de varrido, a partir do qual a distância foi de $10 \mathrm{~m}$. Em algumas ocasiões foi evidenciada variação espacial horizontal de alguns dos principais táxons, bem como da comunidade bentônica dentro de uma escala de $50 \mathrm{~m}$ e $100 \mathrm{~m}$. Esta variação foi provavelmente reflexo da ação das marés meteorológicas que causam abrupta elevação do nível do mar.
\end{abstract}

PALAVRAS-CHAVE. Praias arenosas, comunidade bentônica, distribuição espaço-temporal, riqueza.

Praias arenosas expostas estão entre os ambientes mais dinâmicos, onde as ondas, ventos e marés estão constantemente movimentando o sedimento (MCLACHLAN, 1983). Estes ambientes têm sido caracterizados como fisicamente severos, exibindo heterogeneidade espacial e temporal (Brown \& McLaChLAN, 1990). Esta heterogeneidade está relacionada a fatores físicos, como ação das ondas, tamanho dos grãos de areia e à inclinação da praia (McLachlan, 1983). Além dos efeitos físicos, fatores biológicos como disponibilidade $\mathrm{e}$ busca pelo alimento, efeitos da reprodução na dispersão e assentamento, modos de locomoção e padrões de agregação, competição intra- e interespecífica e efeitos da predação também são responsáveis pela estruturação das comunidades bentônicas de praias arenosas (KNOX, 2000).

NEVEs et al. (2007) estudaram a distribuição da macrofuana através de um gradiente vertical no mesmo local do presente estudo. Diversos estudos foram realizados no Brasil (Souza \& GianuCA, 1995; Borzone \& Souza, 1997; Veloso et al., 1997; Barros et al., 2001; Veloso et al., 2003) e em outras regiões do mundo (Defeo et al., 1992; Jaramillo et al., 1993; Degraer et al., 2003) com o mesmo propósito.

$\mathrm{O}$ aspecto menos abordado nos estudos em praias arenosas é o da distribuição horizontal dos organismos. Este aspecto deve ser considerado, pois é comum a distribuição da macrofauna em manchas (GIMÉNEZ \& YANICELLI, 2000), que podem ser geradas pela combinação de fatores bióticos e abióticos (Defeo \& McLachlan, 2005).
A variação horizontal tem sido documentada para populações e comunidades, sendo atribuída a diversos fatores. Alguns autores identificaram significativa diferença na distribuição das espécies e tamanho dos indivíduos entre as baías e as cristas das cúspides praiais (McLachlan \& Hesp, 1984; James, 1999; Giménez \& YanNicelli, 2000). Schoeman \& Richardson (2002) verificaram que o estado morfodinâmico da praia e interações intraespecíficas regulam a distribuição horizontal do bivalve Donax serra (Röding, 1798). DonN (1987) observou que a abundância de recrutas de $D$. serra foi maior próximo à foz dos rios, porém resultado inverso foi verificado por SCHOEMAN \& RichardSON (2002), que encontraram menor abundância de recrutas de $D$. serra nesses locais. A variação horizontal também pode ser resultante da modificação na granulometria e exposição às ondas (MCLACHLAN, 1996; JAMES \& FAirWEATHER, 1996). GimÉNez \& YANicelli (2000) constataram que a distribuição em manchas de alguns táxons do supralitoral refletiu a quantidade de água contida no sedimento.

A construção de canais de drenagem, exploração comercial do macrozoobentos, engordamento da praia e a construção de barreiras são perturbações antrópicas que influenciam na variabilidade horizontal da macrofauna em praias arenosas (Defeo \& de Alava, 1995; Peterson et $a l .$, 2000; LeRCARI et al., 2002; LeRCARI \& DeFEO, 2003).

Alguns estudos trataram sobre a composição e distribuição vertical do macrozoobentos nos distintos níveis nas praias do extremo sul do Brasil (GianucA, 
1983, 1987; Borzone \& Gianuca, 1990; Neves et al., 2007). Porém, ainda não existem contribuições com o acompanhamento temporal da variação horizontal da macrofauna bentônica nesta região. Desta forma, o presente trabalho tem o objetivo de acompanhar a abundância e a distribuição horizontal do macrozoobentos ao longo de um ano e identificar seus possíveis fatores causais.

\section{MATERIAL E MÉTODOS}

Área de estudo. As praias arenosas do extremo sul do Brasil são expostas, com declividade suave, areia fina, sujeitas à ação de ondas moderadas a fortes, zona de arrebentação bem desenvolvida, apresentando estados morfodinâmicos entre dissipativo e intermediário (Gianuca, 1988; Borzone \& GianucA, 1990; GARCIA \& GiANUCA, 1998). As marés astronômicas são insignificantes, sendo os fatores meteorológicos os principais responsáveis pelas variações do nível da água (CAlliari \& Klein, 1993).

A praia do Cassino, em Rio Grande, RS, apresenta características dissipativas na área próxima aos molhes da barra de Rio Grande, enquanto que no local do presente estudo (Fig. 1), a 17,2 km ao sul do molhe oeste $\left(52^{\circ} 14^{\prime} 040^{\prime \prime} \mathrm{W}\right.$ e $32^{\circ} 15^{\prime} 554^{\prime}$ 'S), a praia apresenta características intermediárias (CALLIARI \& KLEIN, 1993).

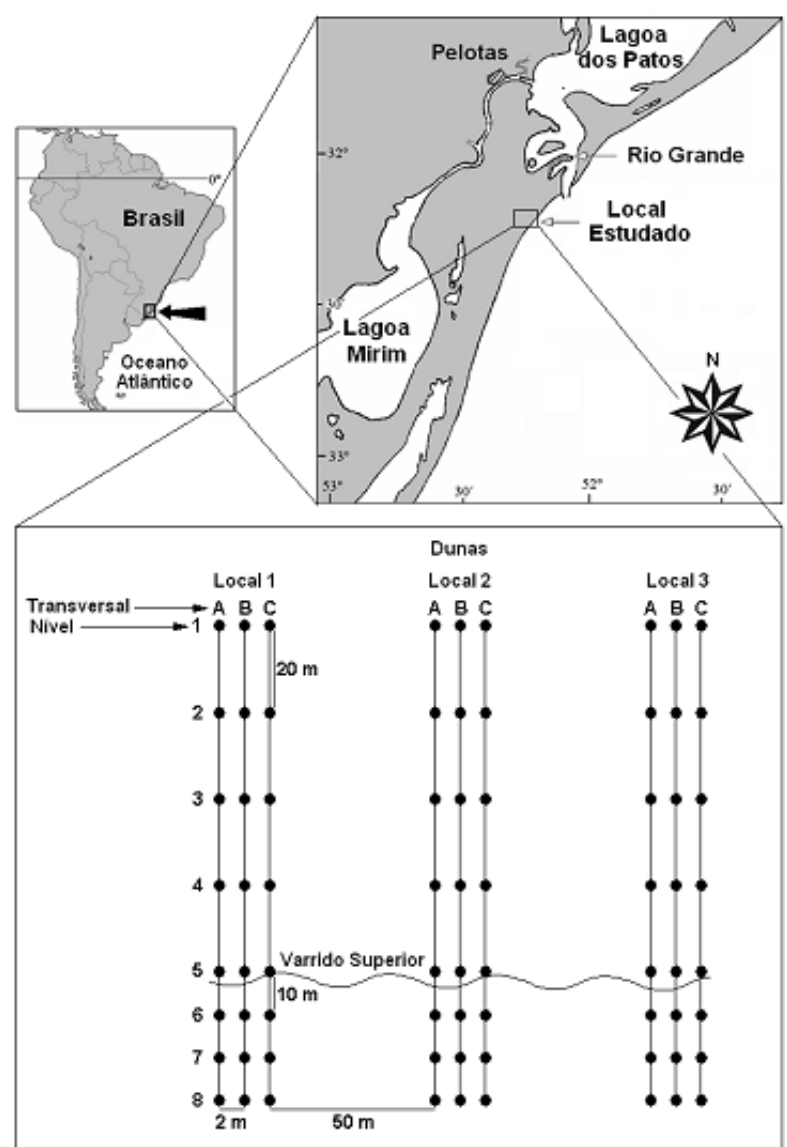

Fig. 1. Localização da área estudada e esquema do desenho amostral a partir do qual foi coletada a macrofauna bentônica no período entre junho de 2004 e maio de 2005, na praia do Cassino, Rio Grande do Sul, Brasil.
Amostragem. As coletas dos macroinvertebrados bentônicos foram realizadas mensalmente durante 12 meses, no período de junho de 2004 a maio de 2005. Foram escolhidos três locais, com 50 m de distância um do outro. Em cada um destes locais foram demarcadas três transversais, 2 metros equidistantes entre si. Cada transversal se estendeu da base das dunas primárias até cerca de $1 \mathrm{~m}$ de profundidade na zona de arrebentação interna, tendo as transversais entre 7 e 8 níveis de coleta. Nas transversais a distância entre os níveis foi de $20 \mathrm{~m}$ até a zona do varrido superior, a partir do qual a distância foi de $10 \mathrm{~m}$ até a isóbata de $1 \mathrm{~m}$ (Fig. 1). A menor distância entre os níveis nas partes inferiores da praia foi devido ao maior número de espécies e organismos que tendem a se concentrar nesta área (GianuCA, 1983; Degraer et al., 2003).

As amostras biológicas foram coletadas utilizandose um tubo extrator de $20 \mathrm{~cm}$ de diâmetro $(0,031416$ $\mathrm{m}^{2}$ ), enterrado no sedimento a $20 \mathrm{~cm}$ de profundidade, pois a maior parte da abundância do macrozoobentos é encontrada nos primeiros $15-20 \mathrm{~cm}$ de profundidade dentro do sedimento (BALlY, 1983). As amostras foram peneiradas em malha de nylon de $0,5 \mathrm{~mm}$ de abertura de poro e o material retido fixado em formalina $10 \%$. Em laboratório, sob estereomicroscópio, os organismos foram quantificados e identificados até o menor nível taxonômico possível.

Para analisar a variação espacial horizontal do macrozoobentos e com o propósito de evitar ruídos causados pela variação espacial vertical, as amostras pertencentes a cada transversal foram somadas mensalmente.

Dados ambientais. Amostras sazonais de sedimento foram tomadas em cada um dos níveis nos três locais. As proporções de areia, silte e argila do sedimento foram determinadas por meio de peneiramento $(>0,062$ $\mathrm{mm}$ de diâmetro) e pipetagem $(<0,062 \mathrm{~mm}$ de diâmetro $)$, conforme Suguio (1973). Mensalmente, durante as coletas, foram registradas a altura (observações visuais) e período médio das ondas (cronômetro digital). Para isso utilizou-se a parte inferior do varrido como referência e o mesmo observador verificou a altura e período de diversas ondas durante as amostragens. Além disso, foi obtida a salinidade (refratômetro ótico), a temperatura do ar e da água (termômetro de mercúrio) em todas as coletas. Dados horários de velocidade e direção do vento e volume de precipitação diária foram fornecidos pela Praticagem da Barra de Rio Grande. Para verificar os efeitos dessas variáveis sobre a macrofauna bentônica levou-se em consideração a quantidade de chuva, intensidade e direção do vento dos sete dias que antecederam as coletas.

Para caracterizar o estado morfodinâmico sazonal da praia, empregou-se o parâmetro adimensional de Dean $\Omega=\mathrm{Hb} / \mathrm{WsT}$, onde $\mathrm{Hb}$ é a altura da onda na arrebentação, Ws é a velocidade de decantação do sedimento e T o período da onda. Valores de $\Omega$ inferiores a 1 representam 
praias reflectivas, praias intermediárias são classificadas no intervalo de 1 a 6 , enquanto praias dissipativas apresentam valores de $\Omega$ superiores a 6 (SHORT \& WrIGHT, 1983). O perfil topográfico da praia foi traçado mensalmente no mesmo local usado para realização das coletas. Para isso foram tomadas medidas desde a base das dunas até o inicio da zona de arrebentação, utilizando-se um nível topográfico.

Análises estatísticas. Com o propósito de analisar a distribuição horizontal do macrozoobentos $(50 \mathrm{~m}$ e $100 \mathrm{~m})$, foram efetuadas análises estatísticas no programa BioEstat versão 4.0. Partindo da soma mensal das amostras de cada transversal, foi verificada a variação horizontal de abundância dos quatro táxons mais representativos dentro de cada mês juntamente com toda comunidade. Para isto utilizou-se o teste nãoparamétrico Kruskal-Wallis com o nível de significância $p<0,05$.

\section{RESULTADOS}

Variáveis ambientais. A salinidade mínima foi registrada em julho e a máxima em janeiro e março. A temperatura da água na arrebentação interna seguiu a mesma tendência da temperatura do ar, sendo as mínimas registradas em julho e maio, e a máxima em janeiro. A altura das ondas apresentou maior valor em novembro, enquanto o período médio das ondas variou entre $8 \mathrm{e}$ 11,9 segundos. Nos sete dias que antecederam as coletas do macrozoobentos, foi observada maior precipitação em outubro (Tab. I)

A partir dos valores do $\Omega$, foi verificado que a praia apresentou estágio do tipo intermediário durante as quatro estações do ano (Tab. II). O tipo de sedimento na maior parte foi classificado como areia fina, porém em algumas ocasiões nas áreas inferiores da praia predominou areia média (Tab. II). A declividade média da praia durante todo período amostral foi de $1,7^{\circ}$, sendo a máxima de $3,3^{\circ}$ observada em fevereiro.

$\mathrm{Na}$ primavera observou-se maior diferença no tamanho de grãos, sendo que a granulometria média foi menor nos locais 2 e 3 quando comparada com o local 1. Nas outras estações do ano essa diferença não foi tão evidente (Fig. 2).

Durante os sete dias que antecederam as coletas, o vento NE foi predominante com velocidade máxima de $26,4 \mathrm{~m} . \mathrm{s}^{-1}$, atingida em outubro. O vento SW foi mais frequente nos períodos de menor temperatura e sua velocidade máxima de $25,1 \mathrm{~m} \cdot \mathrm{s}^{-1}$ ocorreu em março (Tab. III)

Macroinvertebrados bentônicos. Durante os meses de inverno (junho, julho e agosto) 18 táxons foram registrados, sendo Crustacea o grupo com maior número de representantes durante todo período amostral (Tab. IV). A partir da análise dos quatro táxons mais abundantes em cada mês, verificou-se que em junho não houve variação horizontal significativa $(50 \mathrm{~m}$ e 100 m) do anfípode Platyischnopidae (H-2,62; $p-0,27)$, de Donax hanleyanus Philippi, 1847 (H-5,65; $p-0,06)$, do anfípode Phoxocephalopsis sp. (H-0,023; $p-0,99)$, de Euzonus furciferus (Ehlers, 1897) (H-2,05; p-0,36) e da comunidade (H-5,53; $p-0,063)$. A mesma tendência se repetiu no mês de julho com os quatro táxons mais abundante e toda comunidade (Platyischnopidae H-0,022, p-0,99; Mesodesma mactroides Deshayes, 1854 H-3,79, p- 0,15; Phoxocephalopsis sp. H-0,36, p-0,84; Euzonus furciferus $\mathrm{H}-1,11, p-0,57$; comunidade H-1,87, $p$-0,39). Em agosto, novamente, não se detectou variação horizontal expressiva para $D$. hanleyanus (H-4,62; p-0,1), Platyischnopidae (H-3,47; p-0,18), Phoxocephalopsis sp. (H-1,16; p-0,56), Bathyporeiapus sp. (H-5,80; $p-0,055)$ e toda comunidade $(\mathrm{H}-0,87$; $p-0,65)$.

$\mathrm{Na}$ primavera (setembro, outubro e novembro), 21 táxons foram registrados (Tab. IV). Através da análise mensal dos quatro táxons mais abundantes, constatou-se que em setembro não houve variação horizontal significativa de Mesodesma mactroides (H$3,79 ; p-0,15)$, Scolelepis gaucha (Orensanz \& Gianuca, 1974) (H-1,15; $p$-0,56), Platyischnopidae (H-0,83; p-0,66), Bathyporeiapus sp. (H-5,53; p- 0,063) e de toda a comunidade $(\mathrm{H}-1,16 ; p$ - 0,56). Isto também foi constatado em outubro com os quatro principais táxons e toda comunidade (Bathyporeiapus sp. H-5,42, p-0,067; M. mactroides $\mathrm{H}-5,96, p-0,051$; Platyischnopidae $\mathrm{H}-2,76, \quad p-0,25 ;$ D. hanleyanus H- 0,56, p-0,76; comunidade H-5,07, p-0,08). Em novembro Scolelepis gaucha (H-5,96; p-0,051), D. hanleyanus (H-3,29; $p-0,19)$ e toda comunidade (H-5,07; $p-0,079)$ não apresentaram variação horizontal, porém foi detectada variação significativa em $M$. mactroides $(\mathrm{H}-7,2 ; p$ 0,027) e Bathyporeiapus sp. (H- 6,49; p- 0,039).

Ao longo do verão (dezembro, janeiro e fevereiro) registrou-se 21 táxons (Tab. IV). No mês de dezembro não se observou variação horizontal significativa dos quatro táxons mais abundantes, nem da comunidade ( $S$.

Tab. I. Parâmetros abióticos registrados nas coletas mensais da macrofauna bentônica durante um ano, no período de junho de 2004 a maio de 2005, na praia do Cassino, Rio Grande do Sul, Brasil. Os dados de precipitação referem-se ao acumulado durante os sete dias antes da coleta.

\begin{tabular}{|c|c|c|c|c|c|c|c|c|c|c|c|c|}
\hline & Jun & Jul & Ago & Set & Out & Nov & Dez & Jan & Fev & Mar & Abr & Mai \\
\hline Salinidade & 34 & 29 & 34 & 32 & 30 & 31 & 34 & 36 & 34 & 36 & 35 & 32 \\
\hline Temperatura do ar $\left({ }^{\circ} \mathrm{C}\right)$ & 18 & 15 & 17 & 18 & 20 & 24 & 26 & 30 & 22 & 26 & 21,5 & 15 \\
\hline Temperatura da água $\left({ }^{\circ} \mathrm{C}\right)$ & 16 & 14 & 16 & 16 & 20 & 20 & 23 & 26 & 24 & 25,5 & 22 & 13,5 \\
\hline Altura das ondas (m) & - & 1 & 0,75 & 0,5 & 0,75 & 1,5 & 0,5 & 0,75 & 0,75 & 0,5 & 0,5 & 0,75 \\
\hline Período médio das ondas (s) & - & 10 & 8,8 & 10 & 11,8 & 11,9 & 9,7 & 11,7 & 11 & 8 & 10,3 & 11,4 \\
\hline Precipitação (mm) & 8 & 0 & 19,4 & 5 & 53,4 & 12 & 0,6 & 0 & 8 & 12,6 & 23,4 & 7,6 \\
\hline
\end{tabular}


Tab. II. Tamanho médio dos grãos (phi), classificação do sedimento e valor do parâmetro adimensional de Dean $(\Omega)$ sazonalmente no período de junho de 2004 a maio de 2005, na praia do Cassino, Rio Grande do Sul, Brasil.

\begin{tabular}{|c|c|c|c|c|c|c|c|c|}
\hline & \multicolumn{2}{|c|}{ Inverno } & \multicolumn{2}{|c|}{ Primavera } & \multicolumn{2}{|c|}{ Verão } & \multicolumn{2}{|c|}{ Outono } \\
\hline & Média (phi) & Classificação & Média (phi) & Classificação & Média (phi) & Classificação & Média (phi) & Classificação \\
\hline Nível 1 Local 1 & 2,564 & Areia Fina & 2,568 & Areia Fina & 2,764 & Areia Fina & 2,747 & Areia Fina \\
\hline Nível 2 Local 1 & 2,753 & Areia Fina & 2,562 & Areia Fina & 2,723 & Areia Fina & 2,521 & Areia Fina \\
\hline Nível 3 Local 1 & 2,499 & Areia Fina & 2,358 & Areia Fina & 2,508 & Areia Fina & 2,77 & Areia Fina \\
\hline Nível 4 Local 1 & 2,27 & Areia Fina & 2,541 & Areia Fina & 2,546 & Areia Fina & 2,766 & Areia Fina \\
\hline Nível 5 Local 1 & 2,154 & Areia Fina & 2,332 & Areia Fina & 2,383 & Areia Fina & 2,753 & Areia Fina \\
\hline Nível 6 Local 1 & 1,889 & Areia Média & 2,188 & Areia Fina & 2,502 & Areia Fina & 2,465 & Areia Fina \\
\hline Nível 7 Local 1 & 1,774 & Areia Média & 2,214 & Areia Fina & 2,394 & Areia Fina & 2,501 & Areia Fina \\
\hline Nível 8 Local 1 & - & - & - & - & 2,403 & Areia Fina & 2,375 & Areia Fina \\
\hline Nível 1 Local 2 & 2,609 & Areia Fina & 2,499 & Areia Fina & 2,565 & Areia Fina & 2,747 & Areia Fina \\
\hline Nível 2 Local 2 & 2,621 & Areia Fina & 2,691 & Areia Fina & 2,574 & Areia Fina & 2,533 & Areia Fina \\
\hline Nível 3 Local 2 & 2,342 & Areia Fina & 2,765 & Areia Fina & 2,429 & Areia Fina & 2,723 & Areia Fina \\
\hline Nível 4 Local 2 & 2,282 & Areia Fina & 2,53 & Areia Fina & 2,423 & Areia Fina & 2,638 & Areia Fina \\
\hline Nível 5 Local 2 & 2,373 & Areia Fina & 2,363 & Areia Fina & 2,597 & Areia Fina & 2,415 & Areia Fina \\
\hline Nível 6 Local 2 & 1,733 & Areia Média & 2,467 & Areia Fina & 2,198 & Areia Fina & 2,483 & Areia Fina \\
\hline Nível 7 Local 2 & 1,987 & Areia Média & 2,513 & Areia Fina & 2,163 & Areia Fina & 2,098 & Areia Fina \\
\hline Nível 8 Local 2 & - & - & - & - & 1,991 & Areia Média & 2,441 & Areia Fina \\
\hline Nível 1 Local 3 & 2,523 & Areia Fina & 2,755 & Areia Fina & 2,585 & Areia Fina & 2,576 & Areia Fina \\
\hline Nível 2 Local 3 & 2,479 & Areia Fina & 2,748 & Areia Fina & 2,719 & Areia Fina & 2,571 & Areia Fina \\
\hline Nível 3 Local 3 & 2,406 & Areia Fina & 2,787 & Areia Fina & 2,385 & Areia Fina & 2,776 & Areia Fina \\
\hline Nível 4 Local 3 & 2,293 & Areia Fina & 2,774 & Areia Fina & 2,409 & Areia Fina & 2,75 & Areia Fina \\
\hline Nível 5 Local 3 & 2,264 & Areia Fina & 2,766 & Areia Fina & 2,508 & Areia Fina & 2,242 & Areia Fina \\
\hline Nível 6 Local 3 & 1,895 & Areia Média & 2,511 & Areia Fina & 2,37 & Areia Fina & 2,394 & Areia Fina \\
\hline Nível 7 Local 3 & 1,491 & Areia Média & 2,518 & Areia Fina & 2,346 & Areia Fina & 2,442 & Areia Fina \\
\hline Nível 8 Local 3 & - & - & - & - & 1,877 & Areia Média & 2,466 & Areia Fina \\
\hline$\Omega$ & & & & & & & & \\
\hline
\end{tabular}

Tab. III. Característica do vento nos sete dias que antecederam as coletas da macrofauna bentônica realizadas entre junho de 2004 a maio de 2005 , praia do Cassino, Rio Grande do Sul, Brasil.

\begin{tabular}{|c|c|c|c|c|c|c|c|c|c|}
\hline & & $\mathrm{N}$ & $\mathrm{NE}$ & $\mathrm{E}$ & SE & $\mathrm{S}$ & SW & $\mathrm{W}$ & NW \\
\hline \multirow{3}{*}{ Junho } & Frequência (\%) & 7,1 & 32,7 & 0,0 & 0,0 & 0,0 & 9,5 & 29,2 & 21,4 \\
\hline & Velocidade Média $\left(\mathrm{m} . \mathrm{s}^{-1}\right)$ & 3,6 & 5,4 & 0,0 & 0,0 & 0,0 & 6,1 & 5,9 & 3,9 \\
\hline & Velocidade Máxima (m.s- $\left.{ }^{1}\right)$ & 9,8 & 15,6 & 0,0 & 0,0 & 0,0 & 16,1 & 16,6 & 11,2 \\
\hline \multirow{3}{*}{ Julho } & Frequência $(\%)$ & 0,6 & 28,6 & 0,0 & 10,6 & 7,5 & 45,3 & 3,1 & 4,3 \\
\hline & Velocidade Média $\left(\mathrm{m} . \mathrm{s}^{-1}\right)$ & 2,2 & 7,1 & 0,0 & 4,0 & 6,2 & 6,2 & 3,0 & 2,9 \\
\hline & Velocidade Máxima (m.s- $\left.{ }^{1}\right)$ & 4,9 & 16,1 & 0,0 & 6,7 & 12,5 & 13,4 & 8,1 & 5,8 \\
\hline \multirow{3}{*}{ Agosto } & Frequência (\%) & 1,2 & 26,8 & 4,2 & 10,1 & 2,4 & 41,1 & 10,1 & 4,2 \\
\hline & Velocidade Média $\left(\mathrm{m} . \mathrm{s}^{-1}\right)$ & 2,9 & 7,8 & 6,4 & 3,5 & 3,0 & 6,6 & 3,9 & 2,4 \\
\hline & Velocidade Máxima (m.s- $\left.{ }^{1}\right)$ & 6,3 & 19,7 & 9,0 & 7,2 & 6,3 & 15,6 & 9,0 & 4,9 \\
\hline \multirow{3}{*}{ Setembro } & Frequência (\%) & 0,6 & 14,9 & 6,0 & 37,5 & 26,8 & 14,3 & 0,0 & 0,0 \\
\hline & Velocidade Média $\left(\mathrm{m} . \mathrm{s}^{-1}\right)$ & 5,8 & 8,9 & 7,5 & 8,9 & 8,4 & 8,3 & 0,0 & 0,0 \\
\hline & Velocidade Máxima (m.s- $\left.{ }^{1}\right)$ & 12,1 & 16,1 & 12,1 & 17,4 & 17,4 & 16,1 & 0,0 & 0,0 \\
\hline \multirow{3}{*}{ Outubro } & Frequência (\%) & 1,2 & 42,9 & 5,4 & 5,4 & 1,8 & 28,0 & 14,3 & 1,2 \\
\hline & Velocidade Média $\left(\mathrm{m} . \mathrm{s}^{-1}\right)$ & 7,4 & 11,3 & 10,8 & 6,3 & 6,3 & 5,5 & 4,9 & 4,5 \\
\hline & Velocidade Máxima (m.s- $\left.{ }^{1}\right)$ & 14,8 & 26,4 & 17,0 & 12,5 & 9,4 & 13,0 & 9,4 & 8,1 \\
\hline \multirow{3}{*}{ Novembro } & Frequência $(\%)$ & 3,0 & 41,7 & 9,5 & 4,8 & 11,3 & 14,3 & 12,5 & 3,0 \\
\hline & Velocidade Média $\left(\mathrm{m} . \mathrm{s}^{-1}\right)$ & 2,5 & 10,4 & 9,9 & 3,7 & 8,2 & 7,1 & 5,2 & 1,4 \\
\hline & Velocidade Máxima (m.s- $\left.{ }^{1}\right)$ & 7,2 & 21,5 & 15,2 & 7,6 & 23,7 & 24,6 & 13,9 & 4,5 \\
\hline \multirow{3}{*}{ Dezembro } & Frequência $(\%)$ & 0,0 & 18,8 & 37,1 & 44,1 & 0,0 & 0,0 & 0,0 & 0,0 \\
\hline & Velocidade Média $\left({\mathrm{m} . \mathrm{s}^{-1}}^{1}\right)$ & 0,0 & 9,4 & 9,7 & 9,4 & 0,0 & 0,0 & 0,0 & 0,0 \\
\hline & Velocidade Máxima (m.s-1 $)$ & 0,0 & 16,6 & 15,6 & 15,2 & 0,0 & 0,0 & 0,0 & 0,0 \\
\hline \multirow{3}{*}{ Janeiro } & Frequência $(\%)$ & 3,6 & 48,8 & 11,9 & 16,1 & 3,0 & 10,7 & 1,8 & 4,2 \\
\hline & Velocidade Média $\left(\mathrm{m} . \mathrm{s}^{-1}\right)$ & 4,2 & 8,0 & 7,4 & 5,0 & 5,6 & 8,6 & 5,4 & 3,9 \\
\hline & Velocidade Máxima (m.s-1 $\left.{ }^{1}\right)$ & 11,2 & 15,2 & 12,1 & 12,1 & 11,6 & 17,4 & 11,2 & 16,1 \\
\hline \multirow{3}{*}{ Fevereiro } & Frequência $(\%)$ & 4,8 & 28,6 & 18,5 & 20,8 & 6,0 & 17,9 & 1,8 & 1,8 \\
\hline & Velocidade Média $\left(\mathrm{m} . \mathrm{s}^{-1}\right)$ & 4,1 & 10,4 & 11,2 & 7,5 & 6,9 & 6,3 & 4,0 & 3,1 \\
\hline & Velocidade Máxima (m.s- $\left.{ }^{1}\right)$ & 11,6 & 19,2 & 17,0 & 13,9 & 13,4 & 15,6 & 7,6 & 7,2 \\
\hline \multirow{3}{*}{ Março } & Frequência $(\%)$ & 4,8 & 18,5 & 12,5 & 35,7 & 10,1 & 4,2 & 4,8 & 8,9 \\
\hline & Velocidade Média $\left(\mathrm{m} . \mathrm{s}^{-1}\right)$ & 5,3 & 8,5 & 9,6 & 7,0 & 9,2 & 8,1 & 4,2 & 3,5 \\
\hline & Velocidade Máxima (m.s- $\left.{ }^{1}\right)$ & 11,6 & 22,8 & 21,9 & 18,8 & 25,5 & 25,1 & 10,3 & 8,5 \\
\hline \multirow{3}{*}{ Abril } & Frequência $(\%)$ & 6,0 & 23,8 & 5,4 & 11,9 & 10,7 & 20,2 & 6,5 & 15,5 \\
\hline & Velocidade Média $\left(\mathrm{m} . \mathrm{s}^{-1}\right)$ & 2,6 & 9,1 & 8,2 & 9,3 & 7,7 & 5,4 & 3,6 & 5,9 \\
\hline & Velocidade Máxima $\left(\mathrm{m} . \mathrm{s}^{-1}\right)$ & 12,1 & 20,6 & 16,6 & 17,0 & 17,6 & 15,6 & 13,4 & 17,0 \\
\hline \multirow{3}{*}{ Maio } & Frequência (\%) & 3,0 & 16,7 & 4,8 & 5,4 & 1,8 & 28,6 & 24,4 & 15,5 \\
\hline & Velocidade Média $\left(\mathrm{m} . \mathrm{s}^{-1}\right)$ & 4,0 & 4,7 & 2,1 & 5,9 & 6,6 & 6,0 & 5,8 & 4,6 \\
\hline & Velocidade Máxima (m.s- $\left.{ }^{1}\right)$ & 10,3 & 9,4 & 5,8 & 9,4 & 13,0 & 17,9 & 18,8 & 15,6 \\
\hline
\end{tabular}


Tab. IV. Abundância total dos organismos capturados mensalmente na praia do Cassino, Rio Grande do Sul, Brasil no período entre junho de 2004 a maio de 2005. São apresentados também os valores dos índices de diversidade (H') e equitatividade (J') mensais.

\begin{tabular}{|c|c|c|c|c|c|c|c|c|c|c|c|c|c|}
\hline & Jun & Jul & Ago & Set & Out & Nov & Dez & Jan & Fev & Mar & Abr & Mai & Abundância total \\
\hline Scolelepis gaucha (Polychaeta) & 2 & 67 & 243 & 418 & 590 & 65145 & 4461 & 22217 & 2278 & 53 & 7 & 62 & 95543 \\
\hline Euzonus furciferus (Polychaeta) & 52 & 143 & 162 & 99 & 81 & 348 & 32 & 38 & 57 & 35 & 109 & 681 & 1837 \\
\hline Hemipodus olivieri (Polychaeta) & 3 & 12 & 8 & 21 & 27 & 132 & 3 & 1 & 5 & 11 & 15 & 11 & 249 \\
\hline Sigalion cirriferum(Polychaeta) & 5 & 3 & 6 & 5 & 5 & 5 & 4 & 6 & 2 & 4 & 3 & 2 & 50 \\
\hline Capitellidae (Polychaeta) & 0 & 0 & 0 & 0 & 0 & 0 & 2 & 0 & 0 & 0 & 0 & 0 & 2 \\
\hline Capitella sp. (Polychaeta) & 0 & 0 & 0 & 1 & 0 & 0 & 0 & 0 & 0 & 0 & 0 & 0 & 1 \\
\hline Hyalinoecia sp. (Polychaeta) & 0 & 0 & 0 & 0 & 0 & 0 & 0 & 0 & 0 & 1 & 0 & 0 & 1 \\
\hline Hesionidae (Polychaeta) & 0 & 0 & 0 & 0 & 0 & 1 & 0 & 0 & 0 & 0 & 0 & 0 & 1 \\
\hline Grubeulepis bracteata (Polychaeta) & 0 & 0 & 0 & 0 & 1 & 0 & 0 & 0 & 0 & 0 & 0 & 0 & 1 \\
\hline Mesodesma mactroides (Bivalvia) & 33 & 239 & 247 & 764 & 1970 & 748 & 2097 & 9573 & 596 & 660 & 923 & 103 & 17953 \\
\hline Donax hanleyanus (Bivalvia) & 223 & 134 & 479 & 305 & 637 & 492 & 458 & 1339 & 135 & 2472 & 2592 & 433 & 9699 \\
\hline Donax gemmula (Bivalvia) & 0 & 0 & 0 & 0 & 1 & 0 & 28 & 27 & 4 & 895 & 109 & 4 & 1068 \\
\hline Bathyporeiapus sp.(Crustacea) & 33 & 104 & 260 & 321 & 8100 & 724 & 63 & 106 & 133 & 72 & 129 & 60 & 10105 \\
\hline Phoxocephalopsis sp. (Crustacea) & 96 & 179 & 268 & 139 & 586 & 426 & 986 & 452 & 159 & 91 & 131 & 425 & 3938 \\
\hline Platyischnopidae (Crustacea) & 250 & 272 & 350 & 325 & 788 & 314 & 281 & 392 & 112 & 272 & 290 & 224 & 3870 \\
\hline Emerita brasiliensis (Crustacea) & 4 & 14 & 28 & 9 & 9 & 2 & 48 & 736 & 225 & 242 & 2 & 18 & 1337 \\
\hline Excirolana armata (Crustacea) & 33 & 48 & 55 & 68 & 30 & 18 & 81 & 93 & 118 & 37 & 47 & 92 & 720 \\
\hline Macrochiridothea sp. (Crustacea) & 5 & 18 & 11 & 13 & 6 & 16 & 9 & 76 & 15 & 76 & 20 & 26 & 291 \\
\hline Puelche orensansi (Crustacea) & 0 & 0 & 1 & 0 & 5 & 3 & 0 & 4 & 5 & 33 & 4 & 3 & 58 \\
\hline Excirolana braziliensis (Crustacea) & 1 & 0 & 1 & 1 & 1 & 0 & 0 & 0 & 0 & 0 & 0 & 1 & 5 \\
\hline Pinnixa patagoniensis (Crustacea) & 0 & 2 & 0 & 0 & 0 & 0 & 2 & 3 & 0 & 0 & 0 & 0 & 7 \\
\hline Balloniscus sellowii (Crustacea) & 0 & 0 & 1 & 0 & 0 & 0 & 0 & 0 & 0 & 0 & 0 & 0 & 1 \\
\hline Arenaeus cribarius (Crustacea) & 0 & 0 & 0 & 0 & 0 & 0 & 0 & 1 & 0 & 0 & 1 & 0 & 2 \\
\hline Megalope Ocypode quadrata (Crustacea) & 0 & 0 & 0 & 0 & 0 & 0 & 0 & 0 & 0 & 0 & 1 & 0 & 1 \\
\hline Buccinanops duartei (Gastropoda) & 0 & 0 & 0 & 0 & 0 & 0 & 0 & 7 & 1 & 1 & 0 & 0 & 9 \\
\hline Olivancilaria auricularia (Gastropoda) & 1 & 0 & 0 & 1 & 0 & 0 & 0 & 1 & 1 & 1 & 0 & 0 & 5 \\
\hline Nemertea & 6 & 15 & 2 & 5 & 29 & 40 & 44 & 36 & 18 & 13 & 13 & 10 & 231 \\
\hline Oligochaeta & 0 & 0 & 0 & 0 & 0 & 21 & 48 & 22 & 1 & 1 & 1 & 1 & 95 \\
\hline Abundância total mensal & 747 & 1250 & 2122 & 2495 & 12866 & 68435 & 8647 & 35130 & 3865 & 4970 & 4397 & 2156 & \\
\hline Número de táxons & 15 & 14 & 16 & 16 & 17 & 16 & 17 & 20 & 18 & 19 & 18 & 17 & 28 \\
\hline$H^{\prime}(\log e)$ & 1,779 & 2,137 & 2,081 & 1,957 & 1,27 & 0,2877 & 1,42 & 1,033 & 1,482 & 1,624 & 1,351 & 1,903 & 1,299 \\
\hline J' & 0,657 & 0,810 & 0,751 & 0,706 & 0,448 & 0,104 & 0,501 & 0,345 & 0,513 & 0,551 & 0,467 & 0,672 & 0,390 \\
\hline
\end{tabular}

gaucha $\mathrm{H}-4,62$, p-0,1; M. mactroides $\mathrm{H}-2,49$, p-0,29; Phoxocephalopsis sp. H-5,80, p-0,055; D. hanleyanus $\mathrm{H}-5,95, \mathrm{p}-0,051$; comunidade $\mathrm{H}-1,87, p-0,39)$, isto também acorreu em fevereiro (S. gaucha $\mathrm{H}-5,96, p-0,051$;

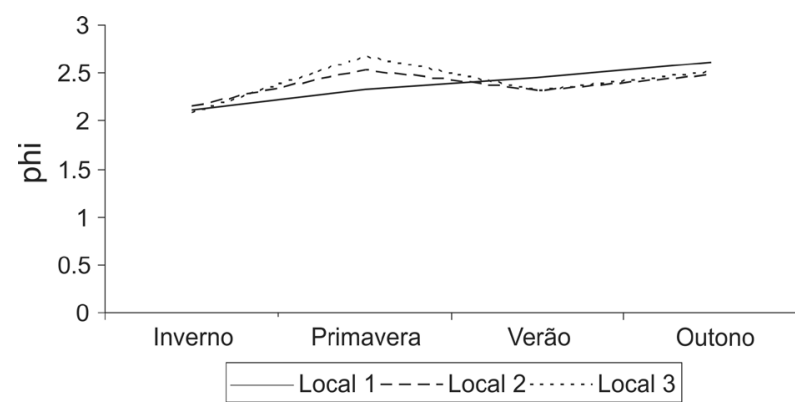

Fig. 2. Variação do tamanho médio dos grãos de areia entre os diferentes locais, nas distintas estações do ano, no período de junho de 2004 a maio de 2005, na praia do Cassino, Rio Grande do Sul, Brasil.
M. mactroides H-5,6, p-0,061; Emerita brasiliensis Schmitt, 1935 H-5,11, p-0,078; Phoxocephalopsis sp. $\mathrm{H}-3,29, p-0,19$; comunidade H-5,07, $p$-0,079). Em janeiro, S. gaucha $(\mathrm{H}-5,96 ; p-0,051), M$. mactroides $(\mathrm{H}-$ $5,96 ; p-0,051)$ e $D$. hanleyanus $(\mathrm{H}-2,40 ; p-0,30)$ não apresentaram variação horizontal, sendo esta verificada em E. brasiliensis $(\mathrm{H}-6,25 ; p-0,044)$ e a comunidade $(\mathrm{H}-$ $7,2 ; p-0,027)$.

Nototal 22 táxons integraram as amostras de outono (março, abril e maio) (Tab. IV). Donax. hanleyanus (H-5,60; p-0,061), Donax gemmula Morrison, 1971 (H-5,60; $p-0,061)$, Platyischnopidae $(\mathrm{H}-4,39 ; p-0,11)$ e a comunidade $(\mathrm{H}-5,42 ; p-0,066)$ não apresentaram variabilidade horizontal significativa em março, mas esta variação foi verificada em M. mactroides (H-6,54; $p$-0,038). Em abril Platyischnopidae (H-7,26; $p-0,026)$ e $M$. mactroides (H-6,49; $p$-0,039) demonstraram 

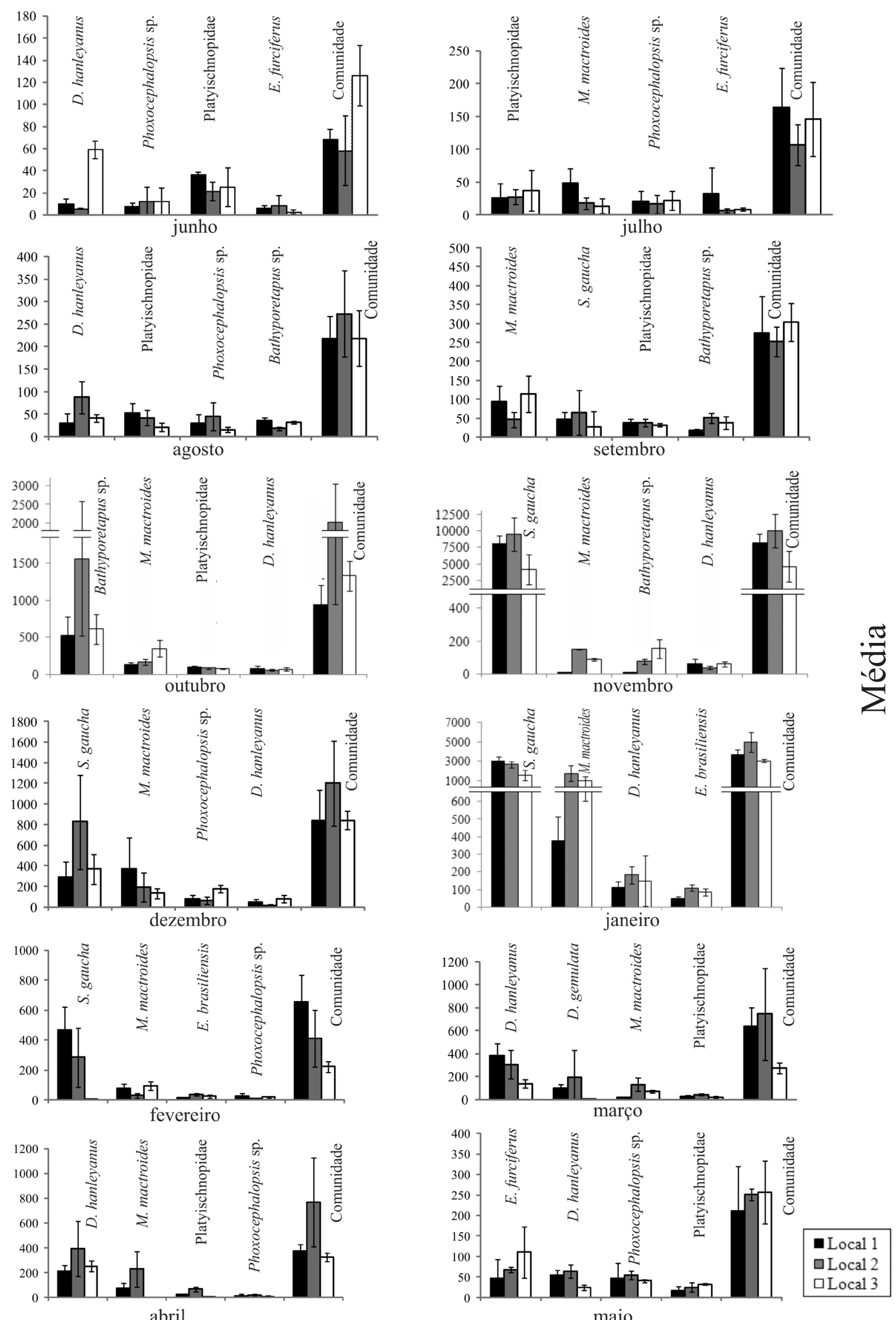

maio

Fig. 3. Média e desvio padrão dos principais táxons nos distintos locais durante os meses que compuseram as coletas (junho de 2004 a maio de 2005), praia do Cassino, Rio Grande do Sul, Brasil. 
variação horizontal, porém não foi constatada em $D$. hanleyanus (H-2,98; p-0,22), Phoxocephalopsis sp. (H$1,88 ; p-0,39)$ e a comunidade (H-5,96; $p$-0,051). Por fim, não foi observada qualquer variação da comunidade e dos quatro táxons mais abundantes em maio (E. furciferus $\mathrm{H}-2,22, \quad p-0,33 ;$ D. hanleyanus $\mathrm{H}-5,96, p-0,051$; Phoxocephalopsis sp. H-2,44, p-0,29; Platyischnopidae $\mathrm{H}-3,82, p-0,15$; comunidade $\mathrm{H}-0,36, p-0,84)$.

Através dos gráficos de média e desvio padrão percebeu-se que há variações marcantes nas médias de alguns táxons entre os locais de coleta em determinados meses. Além disso, nota-se que existe variância importante na média dentro de um mesmo local de coleta (Fig. 3).

\section{DISCUSSÃO}

De acordo com as análises estatísticas, observouse que os organismos mais representativos em cada mês não mostraram variação significativa da distribuição paralela à linha da água na maior parte do estudo.

MCLACHLAN et al. (1996) observaram em uma das praias tropicais de regime macromareal da Austrália, que as espécies apresentaram distribuição em manchas, tanto horizontal quanto verticalmente. Aparentemente em praias de regime macromareal é esperado que interações biológicas tenham um papel mais importante na organização da comunidade, pois a estabilidade do substrato propicia um incremento na quantidade de espécies, consequentemente aumentando a complexidade da comunidade e também as interações entre as espécies (McLachlan et al., 1996). Desta forma, a distribuição da macrofauna bentônica em manchas observada pelos autores acima, pode refletir as características deste tipo de praia, podendo não ocorrer nas praias onde a comunidade é mais controlada por fatores físicos, caso da praia onde ocorreu o presente estudo.

Em novembro, M. mactroides e Bathyporeiapus sp. apresentaram, de acordo com a análise estatística, variação horizontal significativa. Se analisarmos as características dos ventos neste mês, verificaremos que os ventos dos quadrantes sul e sudoeste atingiram altas velocidades. Sabe-se que no local estudado os fatores meteorológicos são os principais responsáveis pelas variações do nível da água (CALliari \& KLEIN, 1993), onde ventos do quadrante sul podem elevar o nível do mar em até $2 \mathrm{~m}$ (BARletta \& CAlliari, 2003). Neste sentido, a maior frequência de ventos sul e sudoeste, atingindo até $23,7 \mathrm{~m} . \mathrm{s}^{-1}$ e $24,6 \mathrm{~m} . \mathrm{s}^{-1}$ respectivamente, deve ter perturbado os padrões de distribuição de ambas populações. Por outro lado, a alta frequência, principalmente dos ventos dos quadrantes sudeste e sul no mês de setembro, provavelmente, não tiveram velocidade suficiente para elevar o nível do mar e, por fim, modificar a distribuição da macrofauna bentônica.

Outubro foi o mês que apresentou maior precipitação, porém este fato não contribuiu para variação horizontal de toda comunidade e das principais populações. Em um estudo conduzido no Uruguai, a descarga de água doce proveniente de um canal artificial afetou significativamente a distribuição horizontal e a abundância da macrofauna bentônica, e a salinidade foi a variável responsável pela variação dos fatores bióticos (LERCARi \& DeFEO, 2003). LERCARI et al. (2002) observaram que os efeitos do canal artificial foram mais notáveis no inverno do que no verão, respectivamente a época de maior e menor precipitação. Além disso, SHOEMAN \& Richardson (2002) registraram na África do Sul que Donax serra apresentou baixa abundância próxima à foz de um rio. Mesmo ao observar maior precipitação, este fato não causou distribuição horizontal diferenciada da macrofauna, pois o local estudado não está sujeito à formação de sangradouros nos períodos de maiores precipitações, nem próximo de sangradouros constantes. Amostragens em locais sob a influência de sangradouros nos períodos de maiores precipitações, ou próximos a sangradouros permanentes, provavelmente, indiquem a ocorrência de variação horizontal do macrozoobentos.

As análises de verão demonstraram que em dezembro e fevereiro não houve variação horizontal significativa dos táxons mais representativos dentro de cada mês, tampouco da comunidade. GIMÉnEZ $\&$ YANNICELLI (2000) verificaram em uma escala de dezenas de metros, em uma praia intermediáriadissipativa uruguaia com declividade de $2,5^{\circ}$ a $8^{\circ}$, que houve diferença significativa na abundância das espécies relacionada ao sistema de cúspides. A mesma tendência foi verificada por BRAZEIRO et al. (1998) em uma praia intermediária de pendente suave no Chile, onde a abundância linear da macrofauna foi significativamente maior nas baías do que nas cristas das cúspides. Na Austrália, McLachlan \& Hesp (1984) notaram que o tamanho dos indivíduos de Hippa australis Hale, 1927 foi significativamente maior nas cristas do que na baía das cúspides, além da preferência dos bivalves Donacilla anugusta, Reeve, 1854 e Donax faba Gmelin, 1791 pela baía da cúspide. Por outro lado, JAmES (1999), em uma praia intermediária da Austrália, não encontrou diferença nas abundâncias de Donax deltoides Lamarck, 1818 entre a baía e crista das cúspides, porém indivíduos menores tenderam a se concentrar nas baías.

No Uruguai as cúspides foram menos conspícuas em uma praia dissipativa com declividade suave e constante ao longo do tempo (média de $2,61^{\circ}$ ) do que em uma praia intermediária com declividade média de $2,65^{\circ}$, chegando a $8^{\circ}$ em determinado período (GiméNEZ \& YANICELli, 1997). No presente estudo a praia foi caracterizada como intermediária, porém com declividade suave (média de $1,7^{\circ}$ ); a declividade máxima foi de $3,3^{\circ}$ registrada em fevereiro. Neste sentido, é esperado que o sistema de cúspides seja menos acentuado neste tipo de praia e, como consequência, 
reduzirá a possibilidade da distribuição da macrofauna ser afetada por este sistema. Por outro lado, a variação horizontal detectada em Emerita brasilienses e em toda a comunidade no mês de janeiro pode refletir as condições ambientais mais amenas do verão. Desta forma, os fatores abióticos serão menos evidentes, fazendo com que as interações biológicas tenham um papel importante na organização de determinadas populações. GimÉNEZ \& YANICELli (2000) observaram, em pequena escala, distribuição espacial agregada ou em manchas de Emerita brasiliensis, sendo maior abundância registrada na baía das cúspides.

Salienta-se que mesmo com maior frequência do vento do quadrante sudeste no mês de dezembro, é provável que a velocidade não foi suficiente para elevar o nível do mar e interferir na distribuição da macrofauna bentônica nesta época do ano.

No outono verificou-se variação horizontal significativa das populações de $M$. mactroides em março e M. mactroides e Platyischnopidae em abril, enquanto em maio nenhuma diferença foi constatada. Um fator que pode ter influenciado o padrão de distribuição horizontal de $M$. mactroides em março foi a elevação do nível do mar. Essa elevação foi proporcionada pelos fortes ventos dos quadrantes sul e sudoeste durante os dias que antecederam a coleta.

Defeo et al. (1992) encontraram significante diferença na estrutura da comunidade bentônica relacionada à granulometria do sedimento de praias uruguaias. McLACHLAN (1996), baseado em uma escala espacial de centenas de metros a quilômetros, concluiu também que houve variação horizontal significativa na estrutura da comunidade bentônica de acordo com o tamanho dos grãos. CALLIARI \& KLEIN (1993) observaram que a variação espacial que ocorre nas praias do extremo sul do Brasil é principalmente devida à ocorrência de biodetritos a aproximadamente $170 \mathrm{~km}$ ao sul do local estudado. Além disso, Figueiredo \& Calliari (2006) verificaram que os sedimentos próximos ao molhe oeste da barra de Rio Grande são mais finos, ocorrendo uma significativa elevação do tamanho médio dos grãos em direção ao sul. Neste contexto, poderíamos esperar encontrar variação horizontal significativa da macrofauna bentônica em uma escala de quilômetros devido à variação no tamanho dos grãos de areia que ocorre nas praias da região. No local estudado, a única época que houve diferença de tamanho de grão um pouco mais evidente foi na primavera. Talvez essa diferença possa ter contribuído, de alguma forma, para a variação horizontal da macrofauna bentônica evidenciada nessa época do ano entre os locais de coleta.

Ao utilizar uma escala de centenas de metros, JAMES \& FAIRWEATHER (1996) registraram, em uma praia arenosa da Austrália, variação da macrofauna bentônica nas partes inferiores da praia, sendo esta relacionada ao gradiente de exposição às ondas. Além disso, também verificaram variação do macrozoobentos no mesolitoral desta praia, porém em escala espacial menor, 40 a 50 m. Dexter (1984) realizou um estudo temporal na Austrália e notou que o número de espécies diminuiu quando a exposição às ondas aumentava. Em direção ao molhe oeste da barra de Rio Grande, ou seja, mais ao norte do local do presente estudo, a energia de onda é notoriamente mais atenuada (Lauro Julio Calliari, com. pess.). Neste local a praia apresenta menor diversidade e abundância de organismos em comparação com a registrada no presente trabalho (SILVA et al., 2008). Devese considerar ainda que essa área sofre maior influência dos molhes da Barra de Rio Grande e encontra-se submetida a periódicos aportes de lama (CALLIARI et al., 2001), os quais também afetam o macrozoobentos (SILVA et al., 2008).

A partir do discutido, verificou-se que em algumas ocasiões foram evidenciadas variação espacial horizontal do macrozoobentos em uma escala de $50 \mathrm{~m}$ e $100 \mathrm{~m}$. Tais variações provavelmente ocorreram devido, principalmente, à ação das marés meteorológicas, em consequência dos fortes ventos sul e sudoeste que antecederam as coletas da macrofauna bentônica em determinados meses.

Ao analisarmos os gráficos de média e desvio padrão, fica evidente que muitos organismos apresentaram diferenças marcantes na distribuição entre os locais. Saliente-se que nesses casos a variação horizontal não ficou evidenciada estatisticamente, pois, muitas vezes, a variância dentro de cada local foi importante, comprovando que a distribuição da macrofauna em manchas, como reportado pela literatura, pode também ocorrer em escala menor da apresentada no presente trabalho.

\section{REFERÊNCIAS BIBLIOGRÁFICAS}

BALLY, R. 1983. Intertidal zonation on sandy beaches of the west coast of South Africa. Cahiers de Biologie Marine 24:85-103.

Barletta, R. C. \& Calliari, L. J. 2003. An assesment of the atmospheric and wave aspects determining beach morphodynamic characteristics along central coast of RS state, southern Brazil. Journal of Coastal Research (special issue) 35:300-308

Barros, F.; Borzone, C. A. \& Rosso, S. 2001. Macroinfauna of six beaches near Guaratuba bay, southern Brazil. Brazilian Archives of Biology and Technology 44:351-364.

Borzone, C. A. \& GianucA, N. M. 1990. A zonação infralitoral em praias arenosas expostas. In: Anais do $2^{\mathbf{0}}$ Simpósio sobre Ecossistemas da Costa Sul e Sudeste Brasileira. São Paulo, ACIESP. v.3, p. 280-287.

Borzone, C. A. \& SouzA, J. R.B. 1997. Estrutura da macrofauna bentônica no supra, meso e infralitoral de uma praia arenosa do sul do Brasil. Oecologia Brasiliensis III:197-212

Brazeiro, A.; Rozbaczylo, N. \& FariñA, J. M. 1998. Distribución espacial de la macrofauna en una playa expuesta de Chile central: efectos de la morfodinámica intermareal. Investigaciones Marinas 26:119-126.

Brown, A. C. \& McLachlan, A. 1990. Ecology of Sandy Shores. Amsterdam, Elsevier. 328p.

Calliari, L. J. \& KLeIn, A. H. F. 1993. Características morfodinâmicas e sedimentológicas das praias oceânicas entre Rio Grande e Chuí, RS. Pesquisas 20:48-56.

Calliari L. J.; Speranski, N. S.; Torronteguy, M. \& Oliveira, M.B. 2001. The mud banks of Cassino beach, southern Brazil: characteristics, processes and effects. Journal of Coastal Research 34:318-325. 
Defeo, O. \& De Alava, A. 1995. Effects of human activities on longterm trends in sandy beach populations: the wedge clam Donax hanleyanus in Uruguay. Marine Ecology Progress Series 123:73-82

Defeo, O.; Jaramillo, E.; \& Lyonnet, A. 1992. Community structure and intertidal zonation of the macroinfauna on the Atlantic coasts of Uruguay. Journal of Coastal Research 8:830-839.

Defeo, O. \& McLachlan, A. 2005. Patterns, processes and regulatory mechanisms in sandy beach macrofauna: a multi-scale analysis. Marine Ecology Progress Series 295:1-20.

Degraer, S.; Volckaert, A. \& Vincx, M. 2003. Macrobenthic zonation patterns along a morphodynamical continuum of macrotidal, low tide bar/rip and ultra-dissipative sandy beaches. Estuarine, Coastal and Shelf Science 56:459-468.

DeXter, D. M. 1984. Temporal and spatial variability in the community structure of the fauna of four sandy beaches in south-eastern. Australian Journal of Marine and Freshwater Research 35(6):663-672.

Donn, T. E. 1987. Longshore distribution of Donax serra in two logspiral bays in the eastern Cape, South Africa. Marine Ecology Progress Series 35:217-222.

Figueiredo, S. A. \& CAlliari, L. J. 2006. Sedimentologia e suas Implicações na Morfodinâmica das Praias Adjacentes às Desembocaduras da Linha de Costa do Rio Grande do Sul. Gravel 4:73-87.

Garcia, V. M. T. \& GianuCA, N. 1998. A praia e a zona de arrebentação. In: Seeliger, U.; Odebrecht, C. \& Castello, J. P. eds. Os ecossistemas costeiro e marinho do extremo sul do Brasil. Rio Grande, Ecoscientia. p.184-189.

GianucA, N. M. 1983. A preliminary account of the ecology of sandy beaches in southern Brazil. In: McLachlan, A. \& ERasmus, T. eds. Sandy Beaches as Ecosystems. The Hague, W. Junk Publishers. p.413-420

1987. Zonação e produção nas praias arenosas do litoral sul e sudeste do Brasil: síntese dos conhecimentos. In: Anais do $1^{\circ}$ Simpósio de Ecossistemas da Costa Sul e Sudeste Brasileira. São Paulo, ACIESP. v.1, p. 313-332.

1988. Recursos naturais das praias arenosas do sul do Brasil. Informes de la UNESCO sobre Ciencias del Mar 47:89-94.

Giménez, L. \& Yannicelli, B. 1997. Variability of zonation patterns in temperate microtidal Uruguayan beaches with different morphodynamic types. Marine Ecology Progress Series 160:197-207.

2000. Longshore patterns of distribution of macroinfauna on a Uruguayan sandy beach: an analysis at different spatial scales and of their pottential causes. Marine Ecology Progress Series 99:11-125

JAMES, R. 1999. Cusps and pipis on a sandy ocean beach in New South Wales. Australian Journal of Ecology 24:587-592.

JAMES, R. J. \& FaIRWEATHER, P. G. 1996. Spatial variation of intertidal macrofauna on a sandy ocean beach in Australia. Estuarine, Coastal and Shelf Science 43:81-107.

Jaramillo, E.; MacLachlan, A. \& Coetezee, P. 1993. Intertidal zonation patterns of macroinfauna over a range of exposed sandy beaches in south-central Chile. Marine Ecology Progress Series, 101:105-117.

Knox ,G. A. 2000. The Ecology of Sea Shores. New York, CRC Press. $555 \mathrm{p}$.

LeRCARI, D. \& DefEo, O. 2003. Variation of a sandy beach macrobenthic community along a human-induced environmental gradient. Estuarine, Coastal and Shelf Science 58:17-24

Lercari, D.; Defeo, O. \& Celentano, E. 2002. Consequences of freshwater canal discharge on the benthic community and its habitat on an exposed sandy beach. Marine Pollution Bulletin 44:1397-1404.

McLachlan, A. 1983. Sandy beaches ecology - a review. In: Mclachlan, A. \& Erasmus, T. eds. Sandy Beaches as Ecosystems. The Hague, W. Junk Publishers. p. 321-380.

.1996. Physical factors in benthic ecology: effects of changing sand particle size on beach fauna. Marine Ecology Progress Series 131:205-217.

McLachlan, A. \& Hesp, P. 1984. Faunal response to morphology and water circulation of a sandy beach with cusps. Marine Ecology Progress Series 19:133-144.

McLachlan A.; Ruyck, A. \& Hacking, N. 1996. Community structure on sandy beaches: patterns of richness and zonation in relation to tide range and latitude. Revista Chilena de Historia Natural 69:451-467.

Neves, L. P.; Silva, P. S. R. \& Bemvenuti, C. M. 2007. Zonation of benthic macrofauna on Cassino beach, southernmost Brazil. Brazilian Journal of Oceanography 55(4):293-307.

Peterson, C. H.; Hickerson D. H. M. \& Johnson, G. G. 2000. Short-term consequences of nourishment and bulldozing on the dominant large invertebrates of a sandy beach. Journal of Coastal Research 16(2):368-378

SCHOEMAN, D. S. \& RichaRdson, A. J. 2002. Investigating biotic and abiotic factors affecting the recruitment of an intertidal clam on an exposed sandy beach using a generalized model. Journal of Experimental Marine Biology and Ecology 276:67-81.

Short, A. D. \& Wright, L. D. 1983. Physical variability of sandy beaches. In: McLachlan, A. \& Erasmus, T. eds. Sandy beaches as ecosystems. The Hague, W. Junk Publishers. p.133-144.

Silva, P. S. R.; Neves, L. P. \& Bemvenuti, C. E. 2008. Temporal variation of sandy beach macrofauna at two sites with distinct environmental conditions on Cassino Beach, extreme southern Brazil. Brazilian Journal of Oceanography 56(4):257-270.

Souza, J. B. R. \& GianucA, N. M. 1995. Zonation and seasonal variation of the intertidal macrofauna on a sandy beach of Paraná State, Brazil. Scientia Marina 59(2):103-111.

Suguio, K. 1973. Introdução à Sedimentologia. São Paulo, EDUSP. $317 \mathrm{p}$.

Veloso, V. G.; Caetano, C. H. S. \& Cardoso, R. S. 2003. Composition, structure and zonation of intertidal macroinfauna in relation to physical factors in microtidal sandy beaches in Rio de Janeiro state, Brazil. Scientia Marina 67:393-402.

Veloso, V. G.; Cardoso, R. S. \& Fonseca, D. B. 1997. Spatio-temporal characterization of intertidal macrofauna at prainha beach (Rio de Janeiro State). Oecologia Brasiliensis III:213-225. 